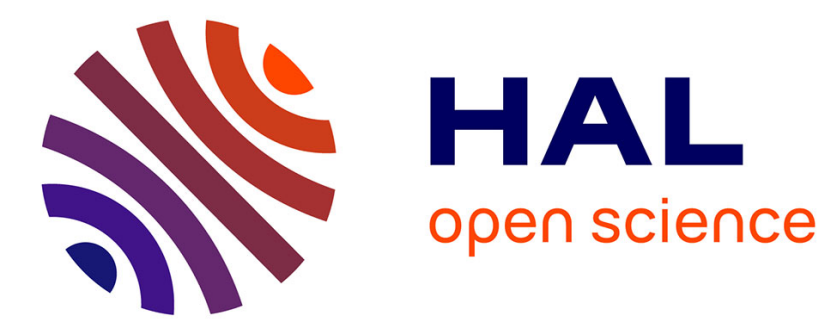

\title{
Multicomponent Seismo-acoustic Surface Waves Beamforming For Undersea Buried Object Detection
}

Cyril Kotenkoff, Jean-Louis Lacoume, Jerome I. Mars

\section{To cite this version:}

Cyril Kotenkoff, Jean-Louis Lacoume, Jerome I. Mars. Multicomponent Seismo-acoustic Surface Waves Beamforming For Undersea Buried Object Detection. IEEE OCEANS 2005, Jun 2005, BREST, France. hal-00376912

\section{HAL Id: hal-00376912 \\ https://hal.science/hal-00376912}

Submitted on 20 Apr 2009

HAL is a multi-disciplinary open access archive for the deposit and dissemination of scientific research documents, whether they are published or not. The documents may come from teaching and research institutions in France or abroad, or from public or private research centers.
L'archive ouverte pluridisciplinaire HAL, est destinée au dépôt et à la diffusion de documents scientifiques de niveau recherche, publiés ou non, émanant des établissements d'enseignement et de recherche français ou étrangers, des laboratoires publics ou privés. 


\title{
Multicomponent Seismo-acoustic Surface Waves Beamforming For Undersea Buried Object Detection
}

\author{
Cyril Kotenkoff, Jean-Louis Lacoume and Jérome Mars \\ Laboratoire des Images et des Signaux \\ 961 rue de la Houille Blanche \\ 38402 Saint Martin d'Hères, France \\ Email: \{cyril.kotenkoff; jean-louis.lacoume; jerome.mars\}@lis.inpg.fr
}

\begin{abstract}
A beamforming method adapted to Stoneley-Scholte waves is presented, in the context of buried objects detection in the seabed. All the waves particularities are exploited in a wideband multicomponent array processing: velocity, dispersion and polarization. These propagation features must be estimated. The incident wave is used for this purpose. Then a signal model is defined according to Stoneley-Scholte wave's propagation, in order to derived optimal and suboptimal receptors. The localization simulations show a good localization behavior for high Signal to Noise Ratios: the performances are close to the asymptotic estimation bounds. For high noise levels, the detection performances depends on the noise spatial coherence. Detection is possible for Signal to Noise Ratios as low as -20dB. Lastly, simulations with Finite Difference signals validate the model and demonstrate that four components are useful in the beamforming.
\end{abstract}

\section{INTRODUCTION}

Seabed surface scanning is an important research topic for military and civil interests. SONAR systems, in particular Synthetic Aperture Sonars, are now widely used for seabed imaging, but are less efficient in case of buried objects. In various papers, the feasibility of detection systems based on seismo-acoustic surface waves has been investigated [1]-[3]. In [1], beamforming results on Stoneley-Scholte waves with a single component array were presented and in [2] detection of scattered Rayleigh waves was performed. We propose here a general multicomponent method employing seismo-acoustic surface waves and working in an unknown sea floor. First we briefly describe the propagation of Stoneley-Scholte waves at the water-sediment interface. Then we present an array processing strategy estimating the dispersion and polarization of these waves and using them in a beamforming method. Lastly detection results for synthetic signals are given and important physical aspects influencing the detection performances are discussed.

\section{Detection System}

\section{A. Stoneley-Scholte waves}

Stoneley-Scholte surface waves (ST waves) propagate at the interface between a fluid and a solid. They combine three wave fields: an acoustic wave in the fluid, a compression wave and a shear wave in the solid. Theses waves decrease vertically at each side of the interface and propagate along the interface at a low velocity. Their polarization in the vertical plane of propagation is elliptic with a vertical long axis. ST waves are dispersive in the sea floor because of velocity heterogeneity in the sediments.

When an explosive source is triggered near the sea floor interface, different kinds of wavefronts occur: compression (P) and shear $(\mathrm{S})$ body waves and surface waves. They have different velocities and polarizations, which make them more or less easy to separate. As illustrated in [3], ST waves are predominant over the others when observed on vertical geophones. Hence these sensors are the best choice for a detection application using ST waves. But in a noisy environment, other components may be useful.

\section{B. System definition}

We propose a detection system exploiting ST waves characteristics. It is composed of a broadband source placed close to the interface and a four component (4C) array of sensors (hydrophones and three axis geophones) on the sea floor. The scanning range is limited by attenuation, increasing with frequency.

\section{ARRAY PROCESSING STRATEGY}

This article presents a localization method based on a classical beamforming $(\mathrm{BF})$ on ST waves. It requires a precise knowledge of the propagation properties in the sediments, otherwise it might be biased. We propose a two steps array processing using a single source emission. The first step is a propagation "learning" from the incident wave. The second is the actual BF method using dispersion and polarization.

\section{A. Learning}

The direct ST wave received on the array is used to estimate the propagation properties. As the wave is predominant on vertical geophones, this single component is used for dispersion estimation. With a linear uniform array, dispersion estimation is performed easily in a wavenumber-frequency representation of the signals. Because of the small number of sensors, it should be interpolated in wavenumber to estimate accurately 
the phase velocity. In case of different geometries, another method must be applied such as multiple filtering [4].

The $4 \mathrm{C}$ polarization vector is the second feature we want to estimate. Precise estimation is difficult because of the guided $S$ waves: they have a velocity close to ST ones and have much more energy in the horizontal components sensors. Moreover, the polarization vector depends on frequency in dispersive media. This leads to choose a unique polarization for all the signal frequencies, but even so, the simulations show a performance increase.

As explained in section IV, the emitted waveform can also be used in the $\mathrm{BF}$, to take into account the source characteristics. It corresponds to any ST signal, of which the propagation delay would been removed. All the sensors signals may be summed to estimate this waveform. Unavoidably, the measure is affected by attenuation.

\section{B. Beamforming}

We scan the sea bottom area, following a grid; for each $\mathrm{BF}$ point, we use the method described in [3], which can be summed up into four steps:

1) the signals are applied a phase velocity correction over the whole propagation distances (source - BF point sensor).

2) corrected signals are summed. When an object exists at the current BF point, the sum is coherent for the echo.

3) a polarization filter gives advantage to ST waves.

4) as the corrected echoes should fit the emitted source waveform, a time matched filtering is applied. It limits the noise contribution to the waveform's time support.

\section{Performance analysis}

In order to study the detection and localization performances, a signal model for ST waves has to be defined. It should include the source spectrum, an attenuation factor rather affecting high frequencies, a propagation delay with phase velocity $c_{S T}(\nu)$ for frequency $\nu$ and a polarization vector $\mathbf{p}_{S T}$. We consider an impulsive pressure source near the interface, with a constant spectrum. The ST response to such a pressure source on a pressure sensor or a velocity sensor is proportional to $\nu^{2.5}$ (see [5] p223 for Rayleigh wave displacements). The frequency dependence of absorption given in [6] is used to define the attenuation factor: at a given distance, it is $a^{-\nu^{1.5}}$ where $a$ depends on the distance and the propagation media. For a reflected wave, an additional attenuation filter is used. It should be high-pass because, buried close to the interface, an object better reflects high frequency ST waves.

In the following, we assume that reflected ST waves have undergone the same attenuation over the whole propagation distance $d$ when received on the sensors. Finally, in the Fourier domain, reflected wave signals (pressure and three velocities) can be written in the compact form:

$$
\left[P v_{z} v_{x} v_{y}\right]^{T}=\mathbf{s}(\nu, \alpha, d)=\mathbf{p}_{S T} s(\nu, \alpha, d)
$$

$$
s(t, \alpha, d) \leftrightarrow s(\nu, \alpha, d)=\alpha(\nu) s(\nu) \exp \left(\frac{-2 \pi j \nu d}{c_{S T}(\nu)}\right)
$$

is the reflected wave signal at a propagation distance $d$ from the source and $s(t) \leftrightarrow s(\nu)$ denotes the ST source signal. The filter $\alpha(\nu)$ represents the reflection coefficient and the exponential factor is the propagation delay.

\section{A. Localization receptor structure}

In [3] we presented a BF method adapted to ST waves. Here we present an analysis from the estimation theory point of view. Let us consider 4C signals received on an array of $n_{s}$ sensors:

$$
\mathbf{r}_{i}(t)=\mathbf{s}\left(t, \alpha, d_{i}\right)+\mathbf{b}_{i}(t)
$$

The subscript $i$ is the sensor index, $d_{i}$ is the total propagation distance from source $\left(x_{s}, y_{s}\right)$ to sensor $\left(x_{i}, y_{i}\right) . \mathbf{s}\left(t, \alpha, d_{i}\right)$ is the 4C ST signal received on sensor $i$ and $\mathbf{b}_{i}(t)$ the $4 \mathrm{C}$ additive noise, independent with the others. In the following, $k$ denotes the component index of a quantity or vector. The noise components are stationary, gaussian, white and independent, with a power spectral density $N_{k}$. For such signals, the maximum likelihood receptor (RML) is [7]:

$$
\int \sum_{i, k} \frac{r_{i k}(t)}{N_{k}} s_{k}\left(t, \alpha, d_{i}\right) d t .
$$

This log-likelihood is either compared to a threshold to detect objects or maximized for precise localization. For high Signal to Noise Ratios (SNR), the localization is asymptotically unbiased and efficient.

When $\alpha$ is unknown, the RML cannot be implemented. It can be simply replaced by the receptor $\mathrm{R} 1$, for which the incident wave signals are used in the correlation:

$$
\int \sum_{i, k} \frac{r_{i k}(t)}{N_{k}} s_{k}\left(t, d_{i}\right) d t .
$$

If the reflection does not introduce any phase difference $(\alpha(\nu)$ real positive), the localization is still asymptotically unbiased. Using Parseval's theorem, we write it in the Fourier domain:

$$
\int \sum_{i, k} \frac{r_{i k}(\nu) p_{S T k}(\nu)}{N_{k}} \exp \left(\frac{2 \pi j \nu d_{i}}{c_{S T}(\nu)}\right) s^{*}(\nu) d \nu .
$$

Superscript * denotes complex conjugation. In this expression appear the BF steps listed in subsection III-B:

1) the exponential factor is the time correction.

2) sum over the sensor index $i$.

3) the sum over $k$ is the projection on $\mathbf{p}_{S T}$.

4) the correlation with $s(\nu)$ is the matched filtering.

The correlation induces secondary maxima in the "likelihood." Let us introduce then the receptor $\mathrm{R} 2$, which is the envelope of R1:

$$
\left|\int \sum_{i, k} \frac{r_{i k}(t)}{N_{k}} \tilde{s}_{k}\left(t, d_{i}\right) d t\right| .
$$

Superscript denotes signal analytical completion. The condition on $\alpha(\nu)$ for unbiased localization becomes a constant 
phase. We show in section $\mathrm{V}$ that both receptors $\mathrm{R} 1$ and $\mathrm{R} 2$ have advantages.

\section{B. Cramer Rao Bounds}

Sea floor positions are located by $x$ and $y$ coordinates. In the Fourier domain, the log-likelihood can be differentiated with respect to them. Two differentiations and the expectation calculation lead to the elements of the Fisher information matrix. For instance, the non-diagonal term is:

$$
J_{12}=\int \sum_{i, k} \frac{1}{N_{k}} \frac{\partial s_{k}\left(\nu, \alpha, d_{i}\right)}{\partial x} \frac{\partial s_{k}^{*}\left(\nu, \alpha, d_{i}\right)}{\partial y} d \nu .
$$

From (1) and (2) the reflected wave components are:

$$
s_{k}\left(\nu, \alpha, d_{i}\right)=p_{S T k}(\nu) \alpha(\nu) s(\nu) \exp \left(\frac{-2 \pi j \nu d}{c_{S T}(\nu)}\right)
$$

We assume that the polarization vectors are constant in the vicinity of the maximum, so that the derivatives only depend on the exponential factor variations. Finally,

$$
J_{12}=\int\left|\frac{\alpha(\nu) s(\nu) 2 \pi \nu}{c_{S T}(\nu)}\right|^{2} \sum_{k} \frac{\left|p_{S T_{k}}(\nu)\right|^{2}}{N_{k}} d \nu \sum_{i} \frac{\partial d_{i}}{\partial x} \frac{\partial d_{i}}{\partial y}
$$

where $d_{i}$ derivatives are (for instance with respect to $x$ ):

$$
\frac{\partial d_{i}}{\partial x}=\frac{\left(x-x_{i}\right)}{\sqrt{\left(x-x_{i}\right)^{2}+\left(y-y_{i}\right)^{2}}}+\frac{\left(x-x_{s}\right)}{\sqrt{\left(x-x_{s}\right)^{2}+\left(y-y_{s}\right)^{2}}} .
$$

Finally the matrix inversion directly provides bounds for the variances along both coordinates $\sigma_{x}^{2}$ and $\sigma_{y}^{2}$ and the asymptotic values for the covariance $\operatorname{cov}_{x y}$.

\section{BEAMFORMING SIMULTATIONS}

We present here simulation results for a linear array. The performances are studied at a broad side position, and a range equal to the array length. The scenario geometry is illustrated in Fig.1.
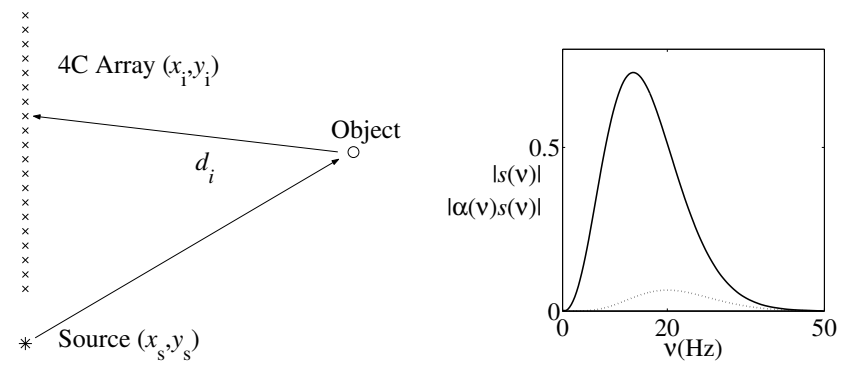

Fig. 1. Scenario geometry with a 20 sensors linear array $(50 \mathrm{~m}$ long, $2.3 \mathrm{~m}$ between sensors), aligned with the source and a buried object $50 \mathrm{~m}$ from the array. Incident (solid line) and reflected (dotted line) spectra; the ordinate unit depends on the signal nature (here the reflected wave has an energy equal to unity).

\section{A. Localization performances}

Simulation results were created using the model in section IV. The source signal was defined as:

$$
s(\nu)=a_{0} \nu^{2.5} a^{-\nu^{1.5}} e^{j \phi(\nu)}
$$

and the high-pass amplitude factor $\alpha(\nu)$ was given the empirical form $\alpha_{0} \nu^{2}$ which roughly fits the amplitude ratio between reflected and emitted waves in our Finite Difference simulations. The constants $a_{0}, a$ and $\alpha_{0}$ were adjusted to normalize the reflected wave in energy, set its spectrum maximum to $20 \mathrm{~Hz}$ and have an amplitude ratio at $20 \mathrm{~Hz}$ equal to 10 (see Fig.1). The source phase $\phi(\nu)$ has no influence on the results.

Fig. 2 shows BF images calculated without noise (ambiguity functions), for the three receptors defined in subsection IVA. The principal lobe in the functions is elongated for the three receptors, leading to a better resolution and precision in range than in bearing. $\mathrm{R} 1$ has a function very similar to RML, apart from slightly larger lobes. R2 has no secondary lobes, its ambiguity function is R1's function envelope. These observations allow to predict the best theoretical performances for the optimal receptor, followed by R1. Even if the reflection is well known, $\mathrm{R} 2$ can be useful for a first rough localization in real applications: when looking for maxima, it does not require

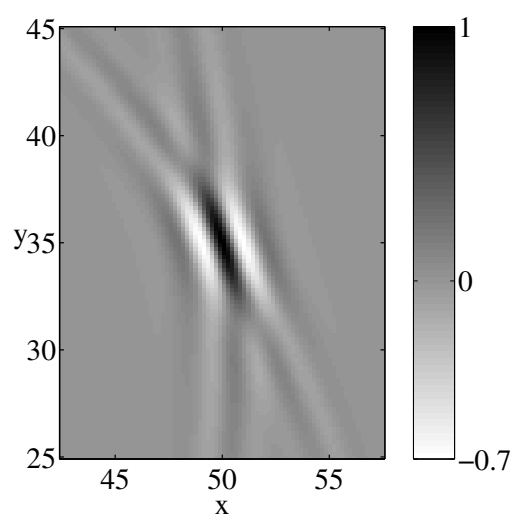

(a)

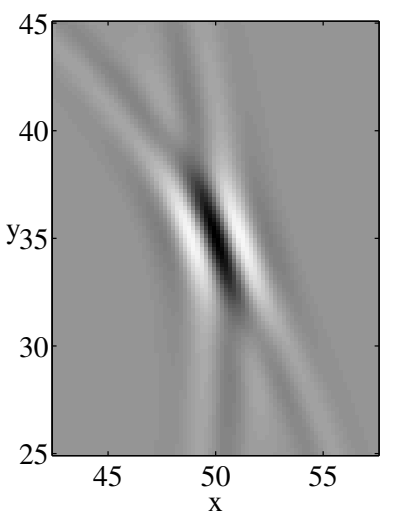

(b)

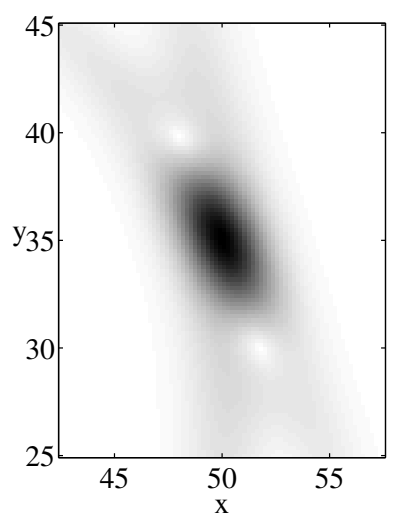

(c)
Fig. 2. Normalized ambiguity functions for RML(a), R1(b) and R2(c), on a 15 by 20 meter area centered around the object. 
an area sampling as dense as R1. R2 Rayleigh resolution was measured without noise $50 \mathrm{~m}$ from the array center, for different object bearings. The $-3 \mathrm{~dB}$ contour line of the ambiguity function has a nearly elliptic shape. The short axis is about $2 \mathrm{~m}$ (i.e. about half the $20 \mathrm{~Hz}$ wavelength) whatever the bearing angle is. It is oriented toward a point between source and array. The long axis is shortest for broadside objects $(4 \mathrm{~m}$, against $10 \mathrm{~m}$ for $70^{\circ}$ off axis objects).

The localization performances were calculated for several SNR. It was defined as the power ratio between $P$ or $v_{z}$ component signal and noise in the signal band. The noise on horizontal velocity components $\left(v_{x}\right.$ and $\left.v_{y}\right)$ was calculated to get the same SNR in average for echoes coming from all directions. One can observe from (6) that when considering a SNR equal for all components, the components signals have the same weights in the beam energy. The estimation variances are shown in Fig.3. The estimated bias were at least ten times smaller than the standard deviations. The Cramer-Rao bounds (CRB) are reached only by the RML, for high SNR. For R1, the asymptotic variation of the three values is only $1.5 \mathrm{~dB}$ higher than the bounds. Using R2 increases the variance along $x\left(\sigma_{x}^{2}\right)$, so that it becomes comparable to $\sigma_{y}^{2}$, as expected from the noiseless observations.

Under a particular SNR threshold, the localization perfor-

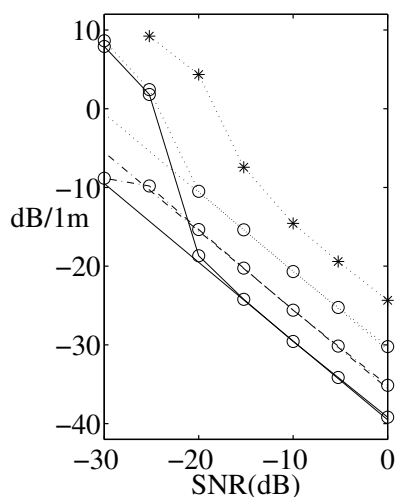

(a)

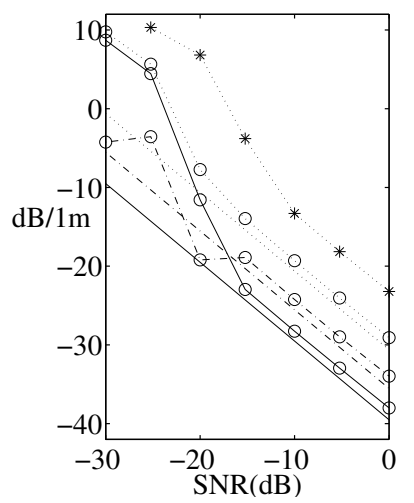

(b)

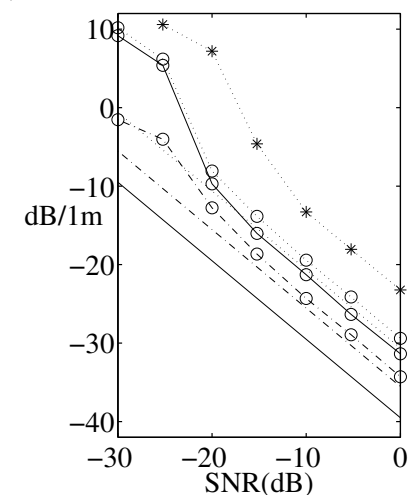

(c)

Fig. 3. Localization variances for RML(a), R1(b) and R2(c). $\sigma_{x}$ (solid), $\sigma_{y}$ (dotted) and $\operatorname{cov}_{x y}^{0.5}$ (dash-dotted) are represented. On each graph, multicomponent estimation variances (o) are compared to CRB (straight lines). Single component estimations are also represented $\left(*\right.$, for $\sigma_{y}$ only). mances decrease rapidly, in fact the variance estimations lose sense: for high noise levels, they increase because more and more "likelihood" maxima are found out of the principal lobe. This amount of false alarms depends on the area where maxima are searched (here a ten by ten meter square centered around the reflector). As the false alarms distribution is uniform, $\sigma_{x}^{2}$ estimation tends to $\sigma_{y}^{2}$,s and $\operatorname{cov}_{x y}$ tends to decrease. In particular, the low value estimated for a $-20 \mathrm{~dB}$ SNR, and receptor $\mathrm{R} 1$, is explained by 21 false alarms only out of 3000 observations.

The 4C Receptors' performances are equivalent to that of corresponding one component receptors for a SNR $6 \mathrm{~dB}$ higher. This measure is consistent with the usual dependence of performances on the sensors number: BF with $N$ times more sensors normally multiplies the output SNR by $\sqrt{N}$.

The small difference in variances between RML and R1 is here an interesting point. But if the spectra of incident and reflected wave happened to be very different, a reflection function could be introduced to get closer to optimal detection. Since the reflection depends on the object size and burying depth, this function would have to be defined a priori.

On Fig.4 are shown detection performances. Receptor Operational Characteristics (ROC) have been calculated for the three receptors and for several SNR. For this SONAR-like application, we found it appropriate to calculate other performance curves. So the false alarm probability was redefined: On graph (d) we consider the probability of at least one

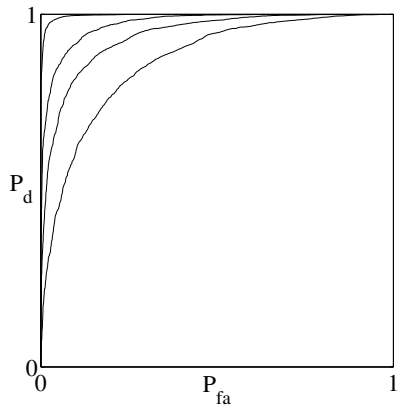

(a)

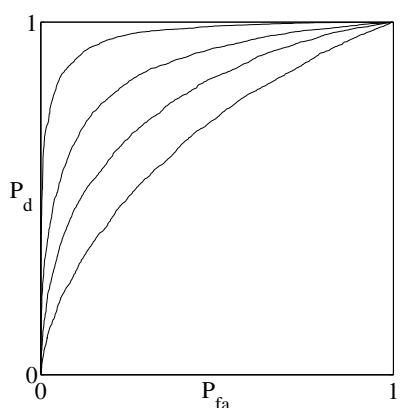

(c)

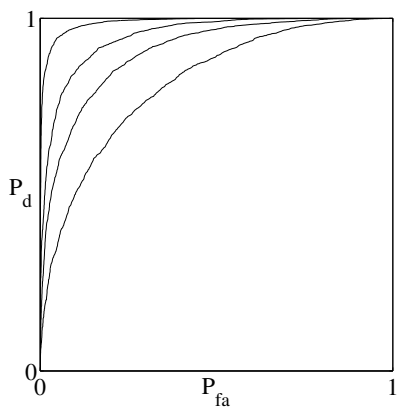

(b)

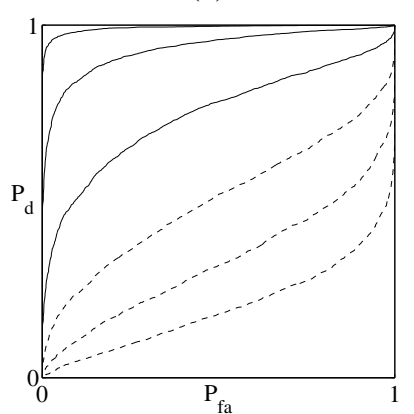

(d)
Fig. 4. Estimated ROC are given for the three receptors RML(a), R1(b) and R2(c), and for different SNR from -25dB (upper curves) down to -33dB (lower curves). On graph (d), the abscissa value is the probability of at least one false alarm in a ten by ten meter square; SNR vary from -20dB (upper curve) to $-30 \mathrm{~dB}$ (lower curve). 
false alarm in a given area. This probability can be higher than the detection probability for high noise levels, as for the dashed curves. Considering a ten by ten meter area, detection is possible for $\mathrm{SNR}$ as low as $-25 \mathrm{~dB}$.

\section{B. Model inaccuracy}

Several factors can deteriorate the performances, such as bad propagation estimation and noise coherence (spatial and intercomponent). The influence of velocity estimation errors on the localization performances was studied. Velocity errors lead to variances increases and localization biases. Localization biases are proportional to the velocity error. As we are dealing with dispersive waves and near-field $\mathrm{BF}$, the maximum beam energy decreases with the velocity error; this trend is illustrated by Fig.5. Decreases are also observed for a bad dispersion estimation, that is when the estimated slope does not fit the real dispersion curve. As a consequence of these phenomena, estimation errors not only deteriorate the localization performances but also the detection ones.

We analyzed the estimation robustness to the noise independence hypothesis. To model inter-component coherence, inter-component correlation coefficients were randomly set (but identically set for all sensors), with a uniform distribution in $[-1,1]$. For spatial coherence, a low-pass spatial filter was applied on the array noise, it was adjusted to get the correlation values measured in [1]: 0.9 at $5 \mathrm{~m}$ and 0.2 at $40 \mathrm{~m}$. Fig.6 illustrates the detection performances. The comparison of these performance curves with the incoherent noise case (Fig.4(d)) indicates that spatial coherence is responsible for most of the performance loss. Considering a ten by ten meter

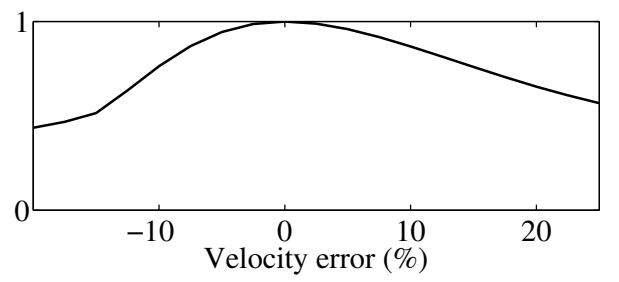

Fig. 5. Variation of the maximum beam energy, calculated without noise.

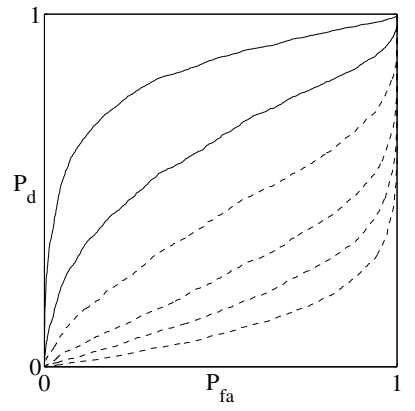

(a)

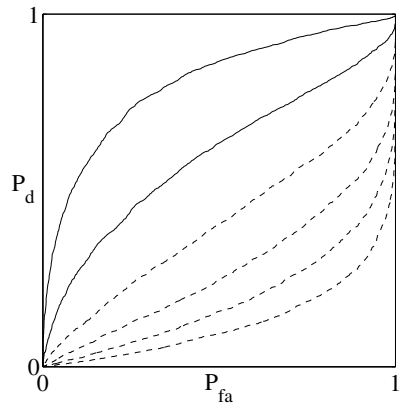

(b)
Fig. 6. Detection performance curves for R2, false alarm probability in a ten by ten meter square, SNR vary from -20dB (upper curves) to -30dB (lower curves). For graph (a) the noise has a spatial coherence only, whereas for graph (b), inter-component coherence has been added.
TABLE I

MEDIA CHARACTERISTICS FOR FD SIMULATION

\begin{tabular}{cccc}
\hline media & $\rho\left(\mathrm{kg} . \mathrm{m}^{-3}\right)$ & $c_{P}\left(\mathrm{~m} . \mathrm{s}^{-1}\right)$ & $c_{S}\left(\mathrm{~m} . \mathrm{s}^{-1}\right)$ \\
\hline \hline water & 1000 & 1500 & \\
\hline sediments (surface) & 1500 & 1500 & 100 \\
\hline sediments (50m depth) & 1500 & 1800 & 500 \\
\hline
\end{tabular}

area, detection is possible for SNR as low as $-20 \mathrm{~dB}$.

\section{Finite Difference signals}

In order to test the $\mathrm{BF}$ method on more realistic cases, synthetic signals were created with a 2D Finite Difference time domain computation of $\mathrm{P}$ and $\mathrm{SV}$ waves [8]. The propagation media are defined on Table I. A buried object is simulated by higher velocities and density in the discrete grid. The pressure and displacements recorded at the interface are interpolated to create a 2D scenario, with body waves and surface waves propagating toward all horizontal directions. We used an impulsive explosive point source near the interface, with a constant spectrum up to $20 \mathrm{~Hz}$ and decaying rapidly beyond. The resulting signal spectra recorded are similar to the synthetic spectra in subsection V-A.

The major difference between these signals and those of the previous subsections is the presence of all kinds of waves, emerging from the object. The noise and SNR definition are the same as in subsection V-A but the body waves' energy is ignored: they do not contribute to the SNR, neither as signals, nor as noise.

For Fig.7, the receptor R2 was applied on noiseless components separately, providing as many $2 \mathrm{D}$ BF images. A small bias in localization distance is observed $(0.85 \mathrm{~m}$, for $x)$; it may be due to the reflection mechanism: the object is not punctual but one meter size in the Finite Difference grid, and the reflection coefficient's phase is not constant in frequency.

Since $\mathrm{P}$ body waves have a very high velocity compared to ST ones, their contribution to the beam energy is poor: images for $P$ and $v_{z}$ are nearly identical. Moreover, they are very similar to R2 ambiguity function represented in Fig.2; this observation gives confidence in the propagation model use in the beamforming. On the contrary, $\mathrm{S}$ waves velocities are barely higher than ST ones. As S waves are predominant on horizontal velocity signals they have a great influence on corresponding BF images; in spite of a different dispersion, they produce strong ghosts. For these simulations, ghosts are still lower than ST detection lobes: hence horizontal components can improve the detection performances at every $\mathrm{BF}$ point. In general cases, BF can be applied with and without horizontal components, when a detection peak is suspected to be a $\mathrm{S}$ detection.

Since these signals have spectra similar to those of the previous subsections, calculating detection curves the same way is not pertinent. It is rather worth defining the correct detection probability versus $\mathrm{S}$ wave false alarm probability. The results are the same as in Fig.4 (a), (b) or (c), with a $3 \mathrm{~dB}$ shift: the difference between ST and S noiseless maxima is 0.69 times smaller than the ST maximum value. 


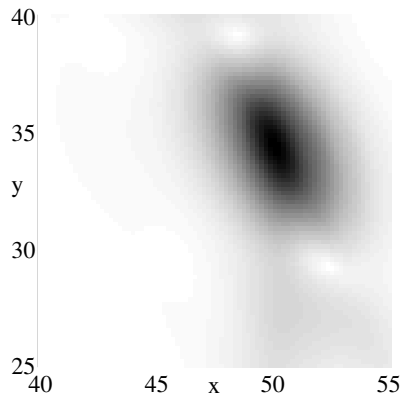

(a)

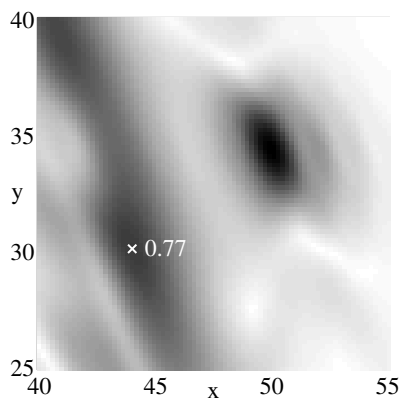

(c)

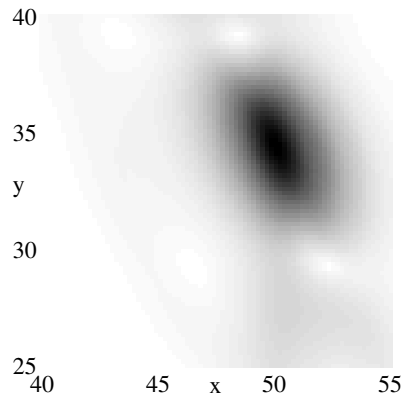

(b)

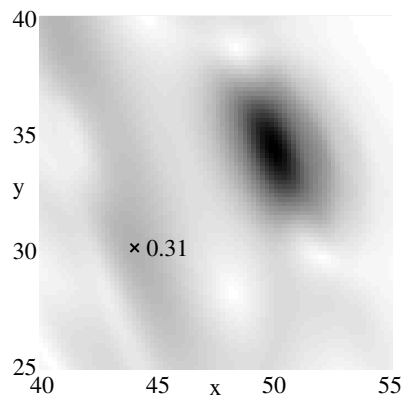

(d)
Fig. 7. Normalized BF images using R2, for single components $P(\mathrm{a})$, $v_{z}(\mathrm{~b})$ and $v_{x}(\mathrm{c})$ and for $4 \mathrm{C}(\mathrm{d})$. The ghosts maxima positions and values are represented. The space coordinates are meters.

\section{CONCLUSION}

A beamforming method adapted to Stoneley-Scholte waves has been presented. All the waves characteristics were considered for the processing. If the dispersion curve in the propagation media is known, a wide band beamforming can be applied, to take advantage of all the signal energy. The polarization of Stoneley-Scholte waves is an important feature: when several sensor components are available (hydrophones and three axis geophones), the different kinds of waves can be identified.

We have shown that beamforming required an accurate estimation of the propagation to avoid performance degradation, such as bias. Hence we proposed to use the incident wave signals for this estimation, afterward precise localization is possible. We defined a signal model according to StoneleyScholte wave's nature and derived optimal and suboptimal receptors. The localization simulations have shown a good localization behavior for high signal to noise ratios: localization variances are close to the asymptotic Cramer Rao Bounds. For high noise levels, the estimated Receptor Operational Characteristics show that detection performance highly depends on the noise spatial coherence, but detection is however possible for Signal to Noise Ratios as low as -20dB. Lastly, simulations were done with Finite Difference computation signals, for a better wave field modeling. The localization method has proved its robustness against other body waves emitted by the object itself and the interest of using four component signals. These signals have also permitted to validate the propagation model of the method.

This method needs to be confronted with realistic noise and signals. In situ experimentation would best provide data for this purpose. Furthermore, detection range should be evaluated for different types of sediments. The localization method could be as well especially adapted to coherent noise and multiple object detection.

\section{REFERENCES}

[1] J. A. TenCate, T. G. Muir, A. Caiti, A. Kristensen, J. F. Manning, J. A. Shooter, R. A. Koch, and E. Michelozzi, "Beamforming on seismic interface waves with an array of geophones on the shallow sea floor," IEEE Journal of Oceanic Engineering, vol. 20, pp. 300-309, October 1995.

[2] E. Smith, P. S. Wilson, F. W. Bacon, J. F. Manning, J. A. Behrens, and T. G. Muir, "Measurement and localization of interface wave reflections from a buried target," J. Acoust. Soc. Am., vol. 103, pp. 2333-2343, May 1998.

[3] C. Kotenkoff, J. L. Lacoume, and J. Mars, "Generation of seismo-acoustic surface waves for undersea buried object detection," in Proc. of the Fourth International Conference on Physics in Signal and Image Processing, vol. 1, (Toulouse, France), pp. 87-91, 2005.

[4] H. A. Pedersen, J. Mars, and P. O. Amblard, "Improving surface wave group velocity measurements by energy reassignment," Geophysics, vol. 62, no. 2, pp. 677-684, 1986.

[5] K. Aki and P. G. Richards, Quantitative seismology. University Science Books, 2002.

[6] T. G. Muir, T. Akal, M. D. Richardson, R. D. Stoll, A. Caiti, and J. M. Hovem, "Comparison of techniques for shear wave velocity and attenuation measurements," in Shear Waves in Marine Sediments (J. M. Hovem, M. D. Richardson, and R. D. Stoll, eds.), pp. 283-294, Kluwer Academic Publishers, 1991.

[7] H. L. Van Trees, Detection, Estimation, and Modulation Theory. part 1, New York: John Wiley \& Sons, 1968.

[8] J. Virieux, "P-sv waves propagation in heterogeneous media: velocitystress finite-difference method," Geophysics, vol. 51, pp. 889-901, 1986. 